17-62). Ethnicity data showed only $37.7 \%$ were white with the majority being Black (54.4\%). $6(7.0 \%)$ of 85 patients had a major NNRTI resistance mutation. Mean nadir CD4 count was 222 (range 5-610). 101 (88.6\%) patients had a CD4 count under 350. 106 reasons for low CD4 count were recorded. 65 patients (64.3\%) had low CD4 counts because of late diagnosis, 15 (14.8\%) had declined ARV when initially offered while 10 (9.9\%) had been lost to followup. The patients starting Rx within 1 year of diagnosis (no=67) had a lower mean nadir CD4 count compared to those diagnosed earlier (no=47) (162 cells vs 271 cells, $p<0.5)$. There was no difference between the two groups in the number of patients having a pretreatment resistance test, the mean CD4 rise 6 months after treatment initiation and the proportion of patients having an undetectable viral load 12 months after treatment initiation. At 6 months the mean CD4 count had risen from 222 at treatment initiation to 360 , but 54 (47.4\%) still had a CD4 count under 350 . The main reasons for this were poor immune recovery in $80.7 \%$, poor adherence $7 \%$, poor attendance $5.3 \%$.

Discussion A proportion of our cohort started ARVs with a low CD4 count mainly due to late diagnosis. This is an important barrier to ARV initiation and needs to be addressed and our audit data would support the need for extra support and resources directed to earlier HIV diagnosis.

\section{P17 FOUR YEARS OF POST EXPOSURE PROPHYLAXIS FOLLOWING SEXUAL EXPOSURE (PEPSE) PRESCRIBING AFTER SEXUAL ASSAULT IN A SEXUAL ASSAULT REFERRAL CENTRE (SARC)}

doi:10.1136/sextrans-2012-050601c.17

\author{
${ }^{1}$ R MacDonald, ${ }^{*}{ }^{2} \mathrm{~T}$ Groom, ${ }^{2} \mathrm{D}$ Wardle. ${ }^{1}$ Sandyford Initiative, Glasgow, UK; ${ }^{2}$ Sandyford \\ Initiative/Archway, Glasgow, UK
}

Aim We have reviewed 4 years of PEPSE use in our SARC and its follow-up and compared with BASHH guidance on PEPSE after sexual assault

Methods Retrospective review of SARC and GUM notes from 12 October 2007 to 12 October 2011

Results 1233 cases seen 127 given PEPSE, for two notes not available Age range $14-55$ years mean $27.81 \%$ were female. $51 \%$ were vulnerable. Ethnicity of assailants, 81 White European, 20 African, 5 Asian, 4 Dark European, 13 unknown. One man had PEPSE twice. Mean time till received PEPSE was $25 \mathrm{~h}$ range $3-168 \mathrm{~h}, 5$ over $72 \mathrm{~h}$. Using BASHH guidelines PEPSE was recommended in $22 \%$, considered in $50 \%$ and $26 \%$ was not recommended as either $>72 \mathrm{~h}$ or low risk exposures. 87 returned at day 3 for review. 29 stopped PEPSE early. One was HIV positive at baseline, 12 due to side-effects, three felt the assault was low risk of HIV at review, 13 for other reasons. $27 \%$ returned for HIV test at 3 months, $14 \%$ at 6 months. No seroconversions seen. 17\% completed PEPSE. 43 given PEPSE while on interacting drugs. Eight were identified and given appropriate management. Most common interaction was hormonal contraception.

Discussion Completion rates for PEPSE were low and similar low rates have been seen in alike studies. No long-term side effects were seen but only $19 \%$ of interactions were identified. PEPSE is a risk reduction method and so clients should not be put at risk of serious drug interactions. 33 were given PEPSE for low risk exposures which is "not recommended" by BASHH. 10 of these accepted full PEPSE course. The decision to start PEPSE is often made under stressful conditions so GUM now review need for PEPSE after completing the starter pack. It is vital staff starting PEPSE prescribe within the guidelines and they and GUM staff consider interactions. We have created a proforma which reminds staff only to give within $72 \mathrm{~h}$ and review interactions. It also outlines HIV risks after exposure and hopefully make it easier to discuss this with the client.

\section{P18 1 POST EXPOSURE PROPHYLAXIS FOLLOWING POSSIBLE EXPOSURE TO HIV INFECTION: AN EVALUATION OF 391 ATTENDANCES AT THREE CENTRAL LONDON SEXUAL HEALTH CLINICS}

doi:10.1136/sextrans-2012-050601c.18

K Janmohamed, ${ }^{*}$ L Bull, D Payne, F Cooper, C Lake, N Nwokolo, M Natha. Chelsea and Westminster NHS Foundation Trust, London, UK

Background Providing post exposure prophylaxis (PEP) following possible HIV exposure is a common GU presentation. However, few studies have evaluated this practice.

Aim(s)/Objective(s) To answer the following on PEP presentations: age, sex, nature of exposure to HIV, time to presentation for PEP, side effects, completion rates, presence of sexually transmitted infections (STIs), appropriateness of PEP dispensing and comparisons of findings with other published studies.

Methods GUM clinic attendances were evaluated from April 2009 to March 2010

Results There were 391 PEP attendances: 373 males (96\% MSM), 18 females. Age range 19-57 (mean 35.4) years. Presentation followed anal sex in $89 \%$, vaginal sex in $5 \%$. The remainder attended following oral sex, splash incidents, injecting drug use, or other exposure. Forty six percent attended within $24 \mathrm{~h}$, in one instance PEP was dispensed beyond $72 \mathrm{~h}$. The majority completed PEP (82\%). GI side effects were experienced by $60 \%$. Baseline screening for hepatitis $\mathrm{B}$ showed active infection in $1 \%$ and immunity in $74 \%$. A baseline HIV test was conducted in all but one patient. An STI screen was conducted at or around day 14 in $69 \%$ of patients, with $12 \%$ testing positive for an STI in line with previously published data. Follow-up rate at 3-6 months was $52 \%$ : Of 203 patients tested for HIV at follow-up, 2 (1\%) tested positive.

Conclusions PEP was dispensed appropriately in the majority of cases. The fact that $82 \%$ of individuals completed treatment despite side effects is likely to be due to the use of more tolerable regimens than were used historically. The presence of an STI in 12\% of people tested highlights the importance of screening in individuals presenting for PEP. The fact that only $52 \%$ of patients attended for a follow-up HIV test at $3-6$ months is of concern and warrants further exploration.

\section{P19 WHAT'S UP? ERECTILE DYSFUNCTION (ED) IN HIV POSITIVE MEN}

doi:10.1136/sextrans-2012-050601c.19

A Williams, ${ }^{*}$ S Dharmaratne, B T Goh. Royal London Hospital, London, UK

Background Antiretroviral therapy in HIV positive patients has resulted in improvements in survival, quality of life and fulfilling sexual relationships. Treatment using phosphodiesterase type 5 inhibitors (PDE5i) for ED has simplified management. However nitrates, including "poppers", and protease inhibitors (PIs) can interact with PDE5i leading to hypotension and high levels of PDE5i. Ethical issues are a consideration as treatments can lead to HIV transmission if safer sex is not practised. We reviewed our HIV positive men with ED and their outcomes after treatment.

Methods 94 HIV positive patients attending our ED clinics from 2006 to 2012 were identified. Data were collected by review of notes and databases. Patients on PIs were started on half of the lowest 
dose tablet of PDE5i and titrated upwards if no response. Safer sex was advised.

Results Of 94 patients, 58 were Caucasian, 34 black; 58 were men who have sex with men, 35 heterosexual; 65 had a stable partner, 41 (47.8\%) had $>30$ lifetime partners, 25 (26.5\%) were $>50$ years; 36 $(38.2 \%)$ used recreational drugs. Two were on therapeutic nitrates. Mean CD4 on presentation was 481 (range 35-1558); 60 (63.8\%) had an undetectable VL at baseline. $30(32 \%)$ had ED symptoms for $>5$ years. Risk factors: smokers 35 (37.2\%); peripheral neuropathy 8 ; diabetic 2; abnormal cholesterol 44 (47\%); abnormal hormone profiles 4 . Sildenafil was the first agent in $55(59 \%)$ patients and tadalafil in $28(30 \%) .18$ were on "poppers" and were told to stop before starting PDE5i. Improvement was noted in 51 (54\%) after the first agent and $68(72 \%)$ after the final agent. 36 (38\%) had PDE5i side effects. 39 (41\%) was on PI based Antiretroviral therapy. None reported priapism.

Conclusion It is safe to treat patients on PI with PDE5i by starting with half of the lowest dose tablet. Treatment of ED improves the quality of life in HIV positive patients but care must be taken to avoid serious drug interaction and safer sex practices should be emphasised.

\section{P20 POTENTIAL IMPACT OF UPDATED UK GUIDELINES FOR USE OF POST EXPOSURE PROPHYLAXIS FOLLOWING SEXUAL EXPOSURE IN A LONDON SEXUAL HEALTH SERVICE}

doi:10.1136/sextrans-2012-050601c.20

${ }^{1} \mathrm{~L}$ Snell, ${ }^{*}{ }^{2} \mathrm{~S}$ G Edwards, ${ }^{2} \mathrm{P}$ D Benn. ${ }^{1}$ UCL Medical School; ${ }^{2}$ Mortimer Market Centre, CNWL NHS FT, London, UK

Background Updated UK guidelines for post exposure prophylaxis following sexual exposure (PEPSE) outline new thresholds for when PEPSE is recommended ( $R$ ), considered (C) or not recommended (NR).

Aim/Objective We compared practice and outcomes according to 2006 and 2011 guidelines.

Methods Retrospective review of electronic patient records between 20 January 2011 and 7 November 2011. Information regarding presentation, recommendations and outcomes were collected. Risk estimates were compared with guidelines. Blood abnormalities were classified grades I-IV. Data were analysed using Microsoft Excel.

Results Of 325 requests to a London sexual health service, PEPSE was issued on 281 occasions to 268 patients. Gender: male $n=258$, female $n=10$, median age: 32 years, sexual orientation: men who have sex with men $n=236$, heterosexual $n=25$, not recorded $n=7$. Risk exposure: unprotected anal $(n=263)$ and vaginal $(n=26)$ intercourse. Source details: HIV $+n=112(40 \%)$, on antiretroviral therapy $\mathrm{n}=31$, viral load known 40 (14\%) (<50 n=26, >50 n=14). $71(26 \%)$ reported taking PEPSE $\geq$ once (range 1-5) previously. $99 \%$ commenced PEPSE within $72 \mathrm{~h}$ (median 30). Comparing those classified as $\mathrm{R}(\mathrm{n}=258)$ and $\mathrm{C}(\mathrm{n}=21)$ according to 2006 guidelines, $27(10 \%)$ were reclassified NR using 2011 guidelines. Completion of 28 days PEPSE was reported in $59 \%$ cases, $100 \%$ adherence in $87 \%$. Eight stopped early due to side effects $(n=4)$ or the source tested HIV- ( $n=4) .148 / 268$ (55\%) had $\geq 1$ blood abnormality, grade I-II $(\mathrm{n}=196)$ and grade III-IV $(\mathrm{n}=29) .1$ patient developed acute interstitial nephritis. 196/268 (73\%) underwent $\geq 1$ screen for sexually transmitted infections; chlamydia $(n=27)$, gonorrhoea $(n=17)$, syphilis $(n=4)$ and hepatitis $B(n=1)$. Of 243 due 4-month followup, $52 \%$ have tested HIV $-(n=122)$ and HIV $+(n=4)$.

Conclusions We report high rates of repeat PEPSE, side effects/blood abnormalities and poor completion rates. Updated guidelines may result in a modest reduction in the use of PEPSE.
POST-EXPOSURE PROPHYLAXIS FOLLOWING SEXUAL ASSAULT

doi:10.1136/sextrans-2012-050601c.21

${ }^{1} \mathrm{R}$ Dhairyawan, ${ }^{*}{ }^{2} \mathrm{~L}$ Muckart, ${ }^{2} \mathrm{G}$ E Forster. ${ }^{1}$ Barts and the London NHS Trust, London, UK; ${ }^{2}$ Haven Whitechapel, London, UK

Background HIV post-exposure prophylaxis (PEP) is recommended for survivors of sexual assault. Completion rates are often lower than for PEP prescribed in other settings, which may be related to psychological issues faced by survivors immediately after the assault and a lower threshold for prescribing.

Aims To study outcomes of survivors of sexual assault prescribed PEP (Truvada and Kaletra) at an inner city sexual assault referral centre (SARC)

Methods Forensic and follow-up notes were interrogated for data on clients prescribed PEP between 1 June 2010 and 31 May 2011.

Results Data were available on 54 clients; 46 were female. Median age was 25 (range 14-40 years). Ethnicity: White European 35/54, 11/54 Black African/Caribbean and 8/54 Asian. 48/54 initiated PEP at the SARC, 4 in A\&E and 2 in sexual health and all within $72 \mathrm{~h}$. Exposure: 37 RVI, 14 RAI, eight unknown. 20/54 had an additional risk: 11 multiple assailants, eight defloration and seven ano-genital trauma. The assailant HIV status was unknown in all cases, but 11 were assessed to be high risk. 16/54 of the clients had never tested for HIV, 14 had tested negative previously and 24 were not documented. All had PEP prescribed within BASHH guidelines (2006). $36 / 54$ continued care at the SARC. $20 / 36(56 \%)$ completed 28 days of PEP. Nine were lost to follow-up, four discontinued due to side effects (Grade 1-2 nausea and vomiting), One due to abnormal blood results (Grade 1 rise in ALT and creatinine), one chose to stop and one was not documented. None had their PEP modified. 13/36 had an HIV test at 3 months post-PEP and all were negative.

Conclusions This study shows that PEP was prescribed within national recommendations. Completion rates were comparable to a local tertiary sexual health/HIV clinic that followed-up patients prescribed PEP after occupational and sexual exposure $(66 \%)$ but lower than the 2006 BASHH standards $(75 \%)$. This suggests that survivors of sexual assault may require greater adherence support.

\section{P22 A USER CENTRED APPROACH TO THE DESIGN OF P-OF-CARE AND SELF-TEST MOBILE PHONE DIAGNOSTICS FOR SEXUALLY TRANSMITTED INFECTIONS (STIS)}

doi:10.1136/sextrans-2012-050601c.22

V Gkatzidou, ${ }^{*}$ K Hone. Brunel University, Middlesex, UK

Background Effective Sexually Transmitted Infection (STI) control is being challenged by inadequate access to prompt diagnosis and treatment for patients and relatively poor community STI surveillance. This work forms part of a larger eSTI ${ }^{2}$ (Electronic Self-Testing Instrument for Sexually Transmitted Infections) consortium developing diagnostic devices for pathogen detection and integrating point-of-care tests with mobile technology.

Aim(s)/Objective(s) Harnessing the widespread mobile phone use, this research develops innovative $\mathrm{eSTI}^{2}$ technologies for reducing STIs transmission and providing greater personal control of sexual health. The aim of this study is to develop a wireless web-based management system that links chlamydia self-test diagnostics to further patient care pathways.

Methods This research adopts a user centred approach to the development of a Human Technology Interface for self-managing STI diagnosis. The research methodology begins primarily with initial exploratory pilot studies to gather functional and user requirements regarding ethical and regulatory requirements of the Human Technology Interface. Iterative development of functional prototypes 